\title{
BMJ Open Implementing post-discharge care following acute kidney injury in England: a single-centre qualitative evaluation
}

\author{
Rebecca Elvey (D) , ${ }^{1,2,3}$ Susan J Howard, ${ }^{2}$ Anne-Marie Martindale (D) , , ${ }^{1,3}$ \\ Thomas Blakeman ${ }^{1,2,3}$
}

To cite: Elvey R, Howard SJ, Martindale A-M, et al. Implementing post-discharge care following acute kidney injury in England: a singlecentre qualitative evaluation. BMJ Open 2020;10:e036077. doi:10.1136/ bmjopen-2019-036077

- Prepublication history and additional material for this paper are available online. To view these files, please visit the journal online (http://dx.doi org/10.1136/bmjopen-2019036077).

Received 03 December 2019 Revised 28 February 2020 Accepted 17 May 2020

Check for updates

C) Author(s) (or their employer(s)) 2020. Re-use permitted under CC BY-NC. No commercial re-use. See rights and permissions. Published by BMJ.

${ }^{1}$ Centre for Primary Care and Health Services Research, Division of Population Health, Health Services Research and Primary Care, School of Health Sciences, The University of Manchester, Manchester, UK ${ }^{2} \mathrm{NIHR}$ Collaboration for Leadership in Applied Health Research and Care (CLAHRC) Greater Manchester, Salford Royal NHS Foundation Trust, Salford, UK

${ }^{3}$ Manchester Academic Health Science Centre (MAHSC),

Manchester, UK

Correspondence to

Dr Rebecca Elvey;

rebecca.elvey@manchester. ac.uk

\section{ABSTRACT}

Objectives We sought to understand the factors influencing the implementation of a primary care intervention to improve post-discharge care following acute kidney injury (AKI).

Design Qualitative study using semi-structured interviews and thematic analysis.

Setting General practices in one Clinical Commissioning Group area in England.

Participants A total of 18 healthcare staff took part in interviews. Participants were practice pharmacists, general practitioners, practice managers and administrators involved in implementing the intervention.

Results We identified three main factors influencing implementation: differentiation of the new intervention from other practice work; development of skill mix and communication across organisations. Overall, post-AKI processes of care were deemed straightforward to embed into existing practice. However, it was also important to separate the intervention from other work in general practice. Dedicating staff time to proactively identify AKI on discharge summaries and to coordinate the provision of care enabled implementation of the intervention. The post-AKI intervention provided an opportunity for practice pharmacists to expand their primary care role. Working in a new setting also brought challenges; time to develop trusting relationships including an understanding of boundaries of clinical expertise influenced pharmacists' roles. Unclear and inconsistent information on discharge summaries contributed to concerns about additional work in primary care.

Conclusions The research highlights challenges around post-discharge management in the primary care context. Coordination and communication were key factors for improving follow-up care following AKI. Further consideration is required to understand patient experiences of the interface between secondary and primary care. The issues pertaining to discharge care following AKI are relevant to practitioners and commissioners as they work to improve transitions of care for vulnerable patient populations.

\section{BACKGROUND}

Ensuring safer transitions of care is a global priority to improve health outcomes. ${ }^{1-4}$ In
Strengths and limitations of this study

- This is the first known study to explore implementation of a post-discharge acute kidney injury intervention in UK primary care.

- The qualitative methodology followed for the process evaluation allowed a deeper understanding of the topic than has previously been available.

- The sample comprised participants who had actively engaged with the intervention, and may therefore represent a more interested viewpoint overall, which should be taken into consideration when interpreting the results.

- Patients did not participate in the study; patient views should be included in future research.

England, recent health policy has placed emphasis on improving the interface between different sectors, with a particular focus on setting 'discharge standards' to improve handover from secondary to primary care. ${ }^{5-7}$ Key requirements are for hospital teams to communicate information about diagnostic tests clearly and in a timely manner and for primary care teams to ensure information is noted promptly and acted on appropriately. ${ }^{8}$

Acute kidney injury (AKI) is a common clinical syndrome that complicates ${ }^{9}$ around $6 \%-8 \%$ of all hospital admissions and is associated with poor health outcomes. ${ }^{10-14}$ As stated within the Kidney Disease Improving Global Outcomes guidelines, it is the interaction between susceptibility and the type and extent of exposure to insults that determines the risk of occurrence of AKI. ${ }^{9}$ Frail older people with multiple long-term conditions and taking multiple medicines are more susceptible to AKI. ${ }^{15}$ In terms of exposure, AKI is often due to the combined effect of infection, fluid loss and medicines that result in reduced perfusion of the kidneys during an acute event (eg, surgery) or acute illness (eg, influenza). ${ }^{915-18}$ 
AKI affects a wide variety of people across a range of hospital wards, and the implications extend beyond the hospital stay. ${ }^{19}$ In the medium to long term, patients who have had an episode of hospital care complicated by AKI are at higher risk of worse health outcomes, including a further episode(s) of AKI, readmission to hospital with prolonged stays, increased development or progression of chronic kidney disease and cardiovascular events and early death. $^{12-14161920}$

There is a pressing need to develop effective interventions to improve outcomes for patients following illness complicated by AKI. ${ }^{19}$ To date, national and international consensus based recommendations emphasise monitoring of kidney function; optimising medicines management and communicating the diagnosis with patients. ${ }^{9} 152122$ For patients who have had an episode of hospital care complicated by AKI, these measures need to be part of the 'transfer of care' from hospital to primary care. However, several gaps in post-discharge care following AKI have been identified. Studies in hospitals in the USA ${ }^{23}$ and France ${ }^{24}$ illustrated that when AKI occurred, it was recorded on less than half of hospital discharge summaries, and evidence from the UK has also shown low levels of accurate recording of AKI in general practice systems. ${ }^{25}$ A US report showed that only three in five patients have their serum creatinine (a main test of kidney function) checked within 90 days of discharge. ${ }^{26}$

In England, recent national patient safety directives and policy drivers have been focused on tackling the adverse outcomes associated with AKI. ${ }^{27-29}$ In 2015-2016, in order to 'develop the knowledge base of GPs (general practitioners)' and to 'positively impact on readmission rates,' NHS England introduced financial incentives to improve discharge care for patients following an episode of illness complicated by AKI. ${ }^{28}$ Payments were made to acute hospital trusts for documentation of four key items within a patient's discharge summary: (1) stage of AKI; (2) evidence of a medicines review having been undertaken; (3) type of blood tests required on discharge and (4) frequency of blood tests required on discharge for monitoring. ${ }^{28}$

Building on the introduction of hospital incentives, we designed, implemented and evaluated a scheme that sought to improve general practice post-discharge care following AKI. ${ }^{28}$ The intervention is described below, the evaluation was a mixed methods study, further details of which including outcomes analysis are reported elsewhere. ${ }^{30}$ The aim of this qualitative evaluation was to explore factors influencing implementation of the primary care intervention, in particular, the context in which the service developed and the experiences of the people providing it.

\section{METHODS}

\section{The intervention}

Following the introduction of hospital-based incentives ${ }^{28}$ we worked in partnership with a local Clinical Commissioning Group (CCG) to design and implement a primary-care based quality improvement intervention to improve general practice post-discharge care following AKI. ${ }^{28} 30$ Incentivised through a local primary care contract, all 31 general practice teams within the CCG were invited to: (1) engage in an audit of post-discharge AKI care; (2) attend a training session about AKI and (3) develop a practice level action plan to improve AKI aftercare. ${ }^{30}$ Aligned with recommended best practice, action plans focused on implementing four key post-discharge processes: (a) recording-AKI diagnosis to be Read coded in primary care; (b) medication review-patient to receive a medication review within 1 month of hospital discharge; (c) monitoring of kidney function-serum creatinine to be checked within 3 months and (d) communication with patients-AKI to be communicated to the patient (and carer). Processes b, c and d also needed to be Read coded. In UK general practice, key clinical information from discharge summaries is entered onto general practice computer systems using 'Read codes' from a coded thesaurus of clinical terms, which is the established way that clinical information is recorded across the NHS. Further details about the intervention are provided in online supplementary table 1 and in the report that has been provided to the funding organisation, which reports on the implementation and our mixed methods evaluation, of which this study was part. ${ }^{30}$

\section{Study design}

A qualitative process evaluation was undertaken. Normalisation process theory (NPT) was used to guide data collection and analysis. ${ }^{31}{ }^{32}$ NPT is a theory of social action that is concerned with understanding the work that people do, individually and collectively, concerning a particular set of practices. NPT has four core concepts which focus on the different kinds of work that people do relating to implementing a new practice: (1) coherence-sensemaking work including understandings of 'what' the new process is and peoples roles in it; (2) cognitive participation-relational work, such as engagement and buy-in to a new process and arranging shared team contributions; (3) collective action-what happens when the new process is operationalised and (4) reflexive monitoringassessments of how things go and the impact it has. ${ }^{31} 33$

\section{Patient and public involvement}

We did not involve patients or the public in this research study.

\section{Sampling and data collection}

The sampling strategy was purposive, being driven by the intervention characteristics and focused on capturing the diversity of experience of those involved in implementation. Accordingly, we sought to recruit people working in clinical, managerial and administrative roles based in a range of GP practices. We gained knowledge of different ways of working through liaison with 'project facilitators' within the project team, who repeatedly visited all practices during the course of the project. Therefore, we had 
insight into different practice approaches to implementation of the intervention.

Once an individual within a practice agreed to participate, we used the snowballing technique to recruit other staff directly involved with the intervention. The aim was to gain greater contextual understanding of the factors surrounding implementation. Potential participants were contacted by email and/or telephone and were provided with information about the purpose and aims of the study. In total, 48 people based in 18 practices were contacted by the research team and invited to take part.

Semi-structured interview topic guides were devised, which included open-ended questions about the intervention, its aims and the context in which it had developed, the participant's role, experiences of delivering the intervention and views about what was working well, what was not working well and possible reasons why. The interviews were audio recorded with participants' consent using digital recorders, and were transcribed by professional transcribers. The software package NVivo V.10 was used to store and manage the data. The interviews were carried out between June and November 2017.

\section{Data analysis}

We explored the implementation process using thematic analysis. ${ }^{34}$ The research team read all the transcripts, using the project research questions as initial themes, as well as the NPT constructs, looking for other emerging categories. Data analysis was an iterative process, with the data read repeatedly; the initial categories were discussed in the team, refined and grouped together into themes. NVivo was used to assign codes to the data, representing the themes.

\section{RESULTS}

A total of 18 people based in eight GP practices participated in interviews; the participating pharmacists (with split roles) also worked across another four practices. The sample comprised practice managers and administrators $(\mathrm{n}=8)$, primary care pharmacists $(\mathrm{n}=5)$ and general practitioners $(n=5)$. The interviews lasted between 16 and $61 \mathrm{~min}$ (median value $=29 \mathrm{~min}$ ); 17 were conducted in person and 1 via the telephone. Thirty people declined invitations to participate, with lack of time being the main reason cited.

Comparative analysis of accounts identified three main factors influencing implementation: differentiation of the new intervention from other practice work; development of skill mix and communication across organisations. We explore each of these in turn and show the range of accounts expressed.

\section{Differentiation of the new intervention from other practice work}

The intervention was deemed to comprise activities that were already part of routine work in general practice. Therefore, its introduction was seen by participants to represent a small change to practice. Rather than introducing substantially new activities, that were new "per se', it involved the reorganisation of familiar tasks into a process which focused on AKI. Before the intervention, practice teams had been recording cases of AKI but the Read coding had not been undertaken consistently; sometimes AKI was not recorded at all on the practice system and when it was coded, the existence of several different codes for AKI and related processes of care added complexity. The work of coding-of the diagnosis of AKI, as well as of the other processes of care-was viewed as essential to the success of the intervention, as without coding, subsequent activity would not be captured in the audit. Practice managers used reminder tools, such as attaching cards with the relevant codes printed on to practice computers: to help prompt correct coding:

We used to do them selectively...but now everyone with a diagnosis of AKI will go through the same process, so it's more organised, more focussed. (GP 04)

Organising care into 'pathways' for groups of patients with particular conditions was a familiar approach for GPs and practice managers who seemed to see the implementation of AKI activity as generally straightforward:

... we do a recall system for most long term conditions, so $[\mathrm{AKI}]$ is another thing where we need to do a follow-up ... we've been sorting [this follow up process] for the last few years so this is just another one added to the list. (practice manager 07 )

Interview participants expressed a need to distinguish AKI-related work as a separate entity or project, in order to ensure the required tasks were undertaken and recorded (ie, Read coded). At most practices, a dedicated individual took responsibility for overseeing the intervention:

I think whereas most tasks would just go back to a generic admin inbox ... there's a specific admin team member that's been identified to take leadership ... I think if you just allow [the project] just to go into the ether to a certain extent it's quite easy to lose the thread. (GP 01)

So, we thought if AKI's were going in there they could be lost in the general. So, what we do, is we just make one pharmacist responsible for it and send them to her. (practice administrator 02)

Pharmacists described working to develop their own ways of working with practice systems, to ensure they proactively searched for patients who needed the processes of care:

Usually I have a system where we've got a search and I can pick those patients up...so that means I have to remember to run the searches every week that I'm here otherwise that patient can...get lost... (practice pharmacist 02) 
The post-AKI intervention represented a subtle change to practice. Dedicating staff time to identify AKI and coordinate the provision of processes of care was deemed helpful to maintain focus on the intervention. To an extent, the post-AKI care processes were straightforward to embed into general practice work. However, differentiating the intervention from other practice work was important. Dedicating staff time to proactively identify AKI on discharge summaries and to coordinate the provision of care to those cases helped enable implementation of the intervention as intended.

\section{Development of skill mix}

Some GPs and practice managers commented on the already busy general practice environment, where workloads were substantial and the AKI intervention was 'another' requirement to accommodate. However, practice teams managed to implement the intervention using a range of approaches to skill mix. As mentioned above, correctly identifying and coding cases of AKI was essential work. Discharge summaries arrive electronically at practices and tend to be accessed first by administrators. At some practices, administrators sent all summaries with an AKI diagnoses to one GP for coding. However, it was notable that the intervention was mainly undertaken by non-GPs. Coding was often undertaken by practice coding teams or by a dedicated administrator.

\section{Developments to pharmacists' roles}

The main change in terms of roles in primary care came through the contributions made by practice pharmacists. As outlined in box 1 , implementation of the post-AKI intervention coincided with an increase in the practice pharmacist workforce in the CCG. Several practice pharmacists had played key roles preparing the practice-level action plans as well as being an information resource within practices. Unsurprisingly, the pharmacists tended to focus on conducting medication reviews. The GP quoted below saw this input from pharmacists as advantageous:

We've got a pharmacist, excellent with medication review. Perhaps, sees some scenarios better than GPs. (GP 03)

Practice pharmacists described how the AKI quality improvement intervention provided opportunities to develop and extend the scope of their work, by allowing them to take a more clinical role. This was largely due to having access to the patients' medical notes, within the practice, a key change for pharmacists who had come into the primary care setting having previously worked in community pharmacy, where they did not have access to patients' medical history:

I think the way I'm dealing with patients with AKI is different...when you come from a community [pharmacy] background you kind of just tend to look at the drugs... what I found with AKI is you kind of have to look at everything...[now] before I ring the patient

\section{Box 1 Practice pharmacists' roles in the UK}

Pharmacists have worked in UK general practices for over 15 years providing of variety of medicine management-related functions, (Silcock) initially in non-patient facing roles but more recently with a greater focus on medicines optimisation and patient-centred care. (Nelson) Around the time that PCM AKI was implemented, there was an increase in the primary care pharmacist workforce in general practices in Bury, with pharmacists employed through various routes, including a national initiative to expand the primary care pharmacy workforce in England (https://www.england.nhs.uk/gp/our-practice-teams/cp-gp/); other pharmacists were directly employed by individual practices. No pharmacists worked full time in any one practice, but split their time between two or more practices.

Silcock J, Raynor DK, Petty D. The organisation and development of primary care pharmacy in the United Kingdom. Health Policy 2004;67(2):207-214.

I do a lot of background reading, read their notes... find out why they were hospitalised... (practice pharmacist 06)

I also get a copy of the discharge letter...so I can conduct a full medication review...it's something that we're doing which is clinical, patient-facing... It's nice to be part of something, to fit into the practice... It's good. (practice pharmacist 02)

While positive examples of successful role expansion and professional development for pharmacists were identified, several challenges were mentioned. Working across different practices, only spending 1 or 2 days at each site sometimes made timely follow-up of patients difficult. Pharmacists were faced with competing demands, for example GPs in a practice could have different priorities from the CCG. Also, as general practice was a new work setting for many practice pharmacists, they were unfamiliar with the systems and routines and needed time to acquaint themselves with new ways of working.

...when we came into the practices we didn't have a role so we don't understand the working pattern of a practice. So I don't understand...who gets the discharge summaries to begin with? ... in GP practice do they go to reception first, do they go to the GP first?... How am I going to get that discharge copy? Because up 'til now the GPs dealt with it, they send it back to reception. How do I fit into that? So I think that was hard work initially. (practice pharmacist 02)

Practice pharmacists had experienced varying reception to their input to general practice teams; some were described as 'very open' in their attitude. At other practices, pharmacists experienced frustrations, for example, they had to conduct medication reviews over the telephone, when they felt face-to-face reviews with patients would be better. The pharmacist quoted below described logistical and relational challenges when trying to conduct patient home visits: 
I think what I will try and do was if the GP was going to do a home visit, like go with them but trying to organise that is just a disaster and the GPs aren't used to somebody going with them... GPs they do just take a lot... of encouragement to change their ways a little bit. I think some of them feel a bit threatened when a pharmacist says, I'll come with you and do this or can I sit in with you on that?... a bit reluctant to do it sometimes. (practice pharmacist 08)

The same pharmacist also cited the limits of her own clinical experience, which restricted what she could accomplish carrying out a medication review:

Also I don't have the experience to be starting and stopping too many medicines. It's still a bit of a development thing for me. (pharmacist 08)

The post-AKI intervention provided an opportunity for practice pharmacists to expand their primary care role. Working in a new setting also brought challenges; time to develop trusting relationships including an understanding of boundaries of clinical expertise influenced pharmacists' roles.

\section{Communication across organisations}

\section{Collaboration within primary care}

Prior to the intervention, practice managers and GPs had little contact with peers in local practices. One practice manager referred to ' 31 different ways of working' across the practices in the CCG. GPs and practice managers had found the training sessions a useful opportunity to learn about the implementation strategies employed at other practices. The approach to training, where practice teams shared details of their action plans, was contrasted with a more common situation whereby staff tended to only communicate with colleagues within their own practice. GPs, managers and administrators had appreciated the, although small, increase in collaboration with other practices that formed part of the AKI intervention. The practice manager quoted below had collaborated with peers in other practices during the course of developing his practice's AKI action plan and had found this to beneficial, but contrasted this with his more general experience of practice relationships:

Practices are generally...not willing to share best practice...I'm trying to do it...managers have the same plates that they're spinning constantly. And if you can share resources that streamlines that work... Practices are not very good at doing it, at all...I find that odd... So if I was struggling with something I would just phone someone...But I know other people won't because they don't want to be perceived as being inferior or whatever...I'm sure there's better action plans than this, but I shared this with five practices before I wrote it down. (practice manager 03)

\section{Communication between primary and secondary care}

Historically, the main form of communication between general practices and hospitals had been via hospital discharge summaries. The standard of communication in these summaries was described as 'hit and miss' and of 'poor quality'. In terms of identifying AKI from the summaries, often a diagnosis of AKI was not highlighted clearly on the first page, making it easy to miss. This was seen as problematic, as transfer of information between secondary and primary care, via hospital discharge summaries, was key to implementing the recommended processes of care following discharge. Some changes (unconnected to this intervention) had been made to the design of the discharge summaries, around the time that the intervention was being implemented. The changes were designed to make an AKI diagnosis easier to notice, for example, the addition of a tick box used to indicate if AKI had occurred. However, GPs and pharmacists still found inconsistencies between information provided on different parts of some discharge summaries:

...it was on the discharge as a diagnosis [of AKI], but further on they've got a box that says, has this patient had AKI? and that said 'no ...The information that comes is still a bit variable. (GP 04)

There are just loads of issues... them being discharged when it says AKI, and then there's no actual document [of a particular test result] and you're thinking, has this been put on by mistake? It doesn't really make sense. (practice pharmacist 08 )

The lack of detail on some summaries meant that even where AKI was specified, several GPs and pharmacists said they needed to look for more information before they were confident that they should Read code a diagnosis of AKI:

...some of these discharge summaries that have come through from hospital... actually when you delved a bit deeper into it, it's [unclear] whether it is an AKI...

So I'm not just going off what we're being told by the hospital because there is a bit of discrepancy in what's coming through... (practice pharmacist 04)

I've got to go back then to have a look...I have to pull the results from the hospital. (GP 04)

Seeking additional information about the patient was one form of activity that took up GP and pharmacist time. As illustrated in the quote below, concern was expressed over the accuracy of information provided. It was felt that attention should be given to ensuring AKI was not being over-diagnosed in hospital, as once made, a diagnosis had downstream consequences for the patient and the practice.

The hospital letters...on AKIs...I'm not sure how accurate they are...So, maybe, that needs tightening up...Because that is often the trigger...we have to start the wheels turning here with all the follow-up 
and the medication... That's quite resource intense, actually... it leads to half a dozen appointments in the surgery... (GP 03)

Communication and collaboration between practices increased during implementation. Unclear and inconsistent information on discharge summaries led to additional work for GPs and pharmacists.

\section{DISCUSSION}

\section{Principal findings}

This study explored factors influencing the implementation of an intervention designed to improve the post-discharge management of AKI in primary care. Our analysis identified three main themes influencing implementation: differentiation of the new intervention from other practice work; development of skill mix and communication across organisations. In the main, GPs and practice managers understood the intervention to involve familiar clinical work with the key changes being the introduction of four recommended processes: diagnostic coding; medication review; kidney monitoring and communication with patients. As is the case in other clinical settings, coordinating a set of tasks in a busy practice environment was challenging. For general practice teams in this study, sharing resources or ideas between practices helped to make the process efficient and having a dedicated lead to coordinate the work within a practice was central. Pharmacists were able to develop their practice roles through contributing to the intervention, although time to develop trusting relationships and understandings of the boundaries of clinical expertise were influencing factors. GPs, managers and administrators had appreciated the, although small, increase in collaboration with other practices that came with implementing the AKI intervention. Communication with secondary care was mainly via discharge summaries, where information about AKI diagnoses was of varying quality; unclear or inconsistent information on summaries led to additional work for GPs and pharmacists.

\section{Strengths and weaknesses of this study}

NPT provided a framework to examine collective work surrounding implementation of the intervention. ${ }^{31-33}$ In particular, the NPT construct 'coherence' was useful in helping to draw out the ways in which participants understood AKI-related care and differentiated it from other practice work. Multiple perspectives on a complex process were gained by interviewing staff with varied primary care roles across different general practices. It was not possible to recruit people who were not providing the intervention, therefore, interviews were undertaken only with people engaged with providing the intervention and therefore may represent a more 'interested' viewpoint, than those who were not engaged with it.
This study focused on the organisational and professional work surrounding post-AKI care. However, it remains unclear what AKI means to patients transitioning between hospital and community settings. Silver et al found that patients with AKI prioritised comorbidities, with 'omission of AKI as part of the ongoing medical history. ${ }^{35}$ Our quantitative findings demonstrated a significant increase in the provision of written information to patients and yet our qualitative evaluation was largely silent on how AKI is being communicated. ${ }^{30}$ It was not part of our original plan to include patients in the study, therefore, their response to the information provided and experiences of communication with primary care professionals about post-AKI care, more generally, remain unknown. Taken together, these limitations mean that research involving patients and carers is a priority in order to better understand how an AKI diagnosis is framed, understood and enacted during transitions of care. ${ }^{36}{ }^{37}$ The mandatory introduction of AKI as a new classification system into the NHS has the potential to 'structure and constrain' care delivery and may confer important consequences for patients. ${ }^{27} 38-41$

\section{Comparison with other studies}

Our qualitative evaluation suggests that managing the demands of implementing post-AKI care processes was achieved through development of practice protocols and delegation of responsibilities to practice staff including practice pharmacists. Previous research has highlighted the centrality of relationship building, including support for staff in new roles and our analysis suggested that AKI-related work helped support integration of practice pharmacists into general practice teams. ${ }^{42-45}$ However, resonating with other recent research, we also identified frustrations and lack of clarity around pharmacists' roles, with limited opportunity to conduct face to face medication reviews. ${ }^{46} 47$

Previous research has confirmed pharmacists' ability to identify and resolve discrepancies in patients' medicines following hospital discharge though there is limited evidence that this leads to a significant reduction in unplanned hospital readmissions. ${ }^{48}$ Though medicines reconciliation is a central component of care following illness complicated by AKI, there is a need to take into account individuals' other health and social needs. ${ }^{49}$ As a key example, AKI in the context of people with heart failure requires particular consideration and delegation to practice pharmacists alone may be inadequate. Studies in Canada and Scotland have shown that acute heart failure is a major cause for the high rates in rehospitalisation following AKI. ${ }^{13}{ }^{14}$ Recent national heart failure guidance emphasises the need to place kidney function results in clinical context and that assessment of a person's fluid status is of greater priority and 'a fundamental goal to improve symptoms and to improve outcome. ${ }^{50} 51$ As expressed by participants in our study, delivery of postacute care following AKI requires the development of 
new skills, a higher intensity multidisciplinary approach and with it the need for a greater clarity of roles and boundaries . ${ }^{52} 53$

\section{Implications for clinicians, policymakers and future research}

As stated by James $e t$ al, 'improved understanding of the current processes of care during follow-up of patients with AKI and of the relationships of these processes with clinical outcomes' is important to 'improve the quality of care and long-term outcomes of patients with AKI. ${ }^{19}$ Our quantitative evaluation indicated that diagnostic coding of AKI in general practice records was associated with improvements in downstream management (medication reviews; kidney monitoring; communication with patients). ${ }^{30}$ Our qualitative findings suggest that allocating a dedicated member of staff to the intervention enabled implementation. Reminder prompts, such as cards with AKI Read codes, around the practice, could help keep an AKI intervention 'in mind' in a busy environment with competing priorities.

In England, the new GP contract specifically seeks to resource the embedding of practice pharmacists, quality improvement and shared learning across practices through the establishment of primary care networks. ${ }^{54}$ This restructuring of care delivery has the potential to address historical limitations in approaches to quality improvement. ${ }^{55-57}$ Though our overall evaluation of the post-AKI improvement intervention demonstrated engagement, there were no observable changes in outcomes. ${ }^{30}$ Evidence of improved coding and management activity does not necessarily equate with improvements in clinical outcomes. Further research is needed to examine interventions that target system factors impacting on patient safety across the interface between primary and secondary care.

Difficulties relating to the variable quality of hospital discharge summaries are well documented in the literature. $^{2324}$ Research has shown that general practice teams experience challenges and additional work to make sense of variable discharge summaries ${ }^{58}$ including when processing information pertaining to AKI. ${ }^{59}$ Furthermore, credibility of data is central to professional engagement in quality improvement activities. ${ }^{60}$ Within this study, GP and pharmacist accounts suggested that work is required to check the accuracy of the AKI diagnosis stated on hospital discharge summaries. This resonates with findings from an ethnographic study examining the implementation of AKI clinical decision support systems within hospital trusts. ${ }^{61}$ Strategies aimed at eradicating under-diagnosis and containing over-diagnosis may help maximise the utility of AKI as a driver of patient safety and reduce unnecessary additional work and downstream noise within the health system. ${ }^{61}{ }^{62}$ The role of AKI nurse specialists to help place AKI alerts in clinical context and then to coordinate safer transitions of care back into the community warrants further examination. ${ }^{61}$

\section{CONCLUSIONS}

Our findings show that post-discharge management of AKI can be aligned with existing work in the primary care team. Dedicating roles and resources to coordinate the multiple components involved in post-discharge care is important. There is potential for practice pharmacists to contribute to post-discharge care, but building trust and understanding of new roles takes time. Further consideration is required to better understand AKI management between secondary and primary care, and to understand patient experiences of this new diagnostic entity. ${ }^{86}$ The issues pertaining to discharge care following AKI are relevant to practitioners and commissioners as they work to improve transitions of care for vulnerable patient populations across multiple healthcare settings.

Twitter Thomas Blakeman @TomBlakeman

Contributors All authors contributed during the planning stage. RE and TB wrote the protocol; A-MM and RE collected the data; RE performed the data analysis; A-MM, TB and SJH supported the analysis. All authors contributed to the final manuscript.

Funding This report is independent research funded by the National Institute for Health Research Collaboration for Leadership in Applied Health Research and Care (NIHR CLAHRC) Greater Manchester and, from $1^{\text {st }}$ October 2019, the NIHR Applied Research Collaboration (ARC) Greater Manchester. The views expressed in this publication are those of the authors and not necessarily those of the National Institute for Health Research or the Department of Health and Social Care.

Competing interests None declared.

Patient and public involvement Patients and/or the public were not involved in the design, or conduct, or reporting, or dissemination plans of this research.

Patient consent for publication Not required.

Ethics approval The study received ethical approval from the Alliance Manchester Business School Panel at The University of Manchester.

Provenance and peer review Not commissioned; externally peer reviewed.

Data availability statement Data sharing not applicable as no datasets generated and/or analysed for this study. No additional data available.

Open access This is an open access article distributed in accordance with the Creative Commons Attribution Non Commercial (CC BY-NC 4.0) license, which permits others to distribute, remix, adapt, build upon this work non-commercially, and license their derivative works on different terms, provided the original work is properly cited, appropriate credit is given, any changes made indicated, and the use is non-commercial. See: http://creativecommons.org/licenses/by-nc/4.0/.

ORCID iDs

Rebecca Elvey http://orcid.org/0000-0003-3754-4979

Anne-Marie Martindale http://orcid.org/0000-0003-1072-3424

\section{REFERENCES}

1 World Health Organisation. Transitions of care: technical series on safer primary care, 2016. Available: https://apps.who.int/ iris/bitstream/handle/10665/252272/9789241511599-eng.pdf? sequence $=1$ [Accessed 15 Oct 2019].

2 Couturier B, Carrat F, Hejblum G. A systematic review on the effect of the organisation of hospital discharge on patient health outcomes. BMJ Open 2016;6:e012287.

3 National Institute for Health and Care Excellence. Discharge from hospital to a community or care home setting for adults with identified social care needs, 2017.

4 National Institute for Health and Care Excellence. Transition between inpatient hospital settings and community or care home settings for adults with social care needs, NICE guideline full version, 2015. https://www.nice.org.uk/guidance/ng27/evidence/full-guideline-pdf2185185565 
5 Department of Health. Five year forward view, 2014.

6 NHS England. The NHS long term plan. England: NHS, 2019.

7 Department of Health. Transforming primary care, 2014.

8 NHS England Patient Safety Domain. Standards for the communication of patient diagnostic test results on discharge from hospital, 2016.

9 Kidney Disease Improving Global Outcomes (KDIGO) Acute Kidney Injury Work Group. KDIGO clinical practice guideline for acute kidney injury. Kidney Int Suppl 2012;2:1-138.

10 Selby NM, Kolhe NV, McIntyre CW, et al. Defining the cause of death in hospitalised patients with acute kidney injury. PLOS One 2012;7:e48580.

11 Selby NM, Casula A, Lamming L, et al. An organizational-level program of intervention for AKI: a pragmatic stepped wedge cluster randomized trial. J Am Soc Nephrol 2019;30:505-15.

12 Wonnacott A, Meran S, Amphlett B, et al. Epidemiology and outcomes in community-acquired versus hospital-acquired AKI. Clin J Am Soc Nephrol 2014;9:1007-14.

13 Silver SA, Harel Z, McArthur E, et al. 30-day readmissions after an acute kidney injury hospitalization. Am J Med 2017:130:163-72.

14 Sawhney S, Marks A, Fluck N, et al. Acute kidney injury as an independent risk factor for unplanned 90-day hospital readmissions. BMC Nephrol 2017;18:9.

15 National Institute for Health and Care Excellence. Acute kidney injury: prevention, detection and management. Clinical guideline [CG169]. NICE, 2013.

16 Think Kidneys. Acute kidney injury best practice guidance: responding to AKI warning stage test results for adults in primary care, 2018.

17 Liaño F, Pascual J. Epidemiology of acute renal failure: a prospective, multicenter, community-based study. Kidney Int 1996;50:811-8.

18 Feehally J, Gilmore I, Barasi S, et al. RCPE UK consensus conference statement: management of acute kidney injury: the role of fluids, e-alerts and biomarkers. J R Coll Physicians Edinb 2013;43:36-8.

19 James MT, Bhatt M, Pannu N, et al. Long-term outcomes of acute kidney injury and strategies for improved care. Nat Rev Nephrol 2020;16:193-205.

20 National Confidential Enquiry into Patient Outcome and Death. Adding insult to injury, 2009. https://www.ncepod.org.uk/ 2009report1/Downloads/AKI_report.pdf

21 National Institute for Health and Care Excellence. Acute kidney injury: quality standard [QS76]. NICE, 2014.

22 Silver SA, Adu D, Agarwal S, et al. Strategies to enhance rehabilitation after acute kidney injury in the developing world. Kidney Int Rep2017;2:579-93.

23 Greer RC, Liu Y, Crews DC, et al. Hospital discharge communications during care transitions for patients with acute kidney injury: a cross-sectional study. BMC Health Serv Res 2016;16:449.

24 Sautenet B, Caille A, Giraudeau B, et al. Deficits in information transfer between hospital-based and primary-care physicians, the case of kidney disease: a cross-sectional study. J Nephrol 2015;28:563-70.

25 University of Birmingham and University of York Health Economics Consortium (NCCID). Development feedback report on piloted indicators QOF indicator area: acute kidney injury (AKI), 2017. https:// www.nice.org.uk/Media/Default/Standards-and-indicators/QOF\% 20Indicator\%20Key\%20documents/NM152\%20piloting\%20report. pdf

26 Saran R, Li Y, Robinson B, et al. US renal data system 2015 annual data report: epidemiology of kidney disease in the United States. Am $J$ Kidney Dis 2016;67:A7-8.

27 NHS England. Patient safety alert: stage 3 directive: standardising the early identification of acute kidney injury. England: NHS, 2014. https://www.england.nhs.uk/patientsafety/wp-content/uploads/sites/ 32/2014/06/psa-aki2.pdf

28 NHS England Contracting and Incentives Team. Commissioning for quality and innovation (CQUIN) guidance 2015/16, 2015. https:// www.england.nhs.uk/wp-content/uploads/2015/03/9-cquin-guid2015-16.pdf

29 Think Kidneys. Discharge summaries for patients whose hospital admission included an episode of AKI: minimum data content, 2016.

30 NIHR Collaboration for Leadership in Applied Health Research and Care (Greater Manchester). Primary care management of acute kidney injury in bury CCG, 2019. https://www.arc-gm.nihr.ac.uk/ projects/bury-post-aki

31 May CR, Mair F, Finch T, et al. Development of a theory of implementation and integration: normalization process theory. Implement Sci 2009;4:29.
32 Murray E, Treweek S, Pope C, et al. Normalisation process theory: a framework for developing, evaluating and implementing complex interventions. BMC Med 2010;8:63.

33 May C, Finch T, Mair F, et al. Understanding the implementation of complex interventions in health care: the normalization process model. BMC Health Serv Res 2007;7:48.

34 Silverman S. Interpreting qualitative data: methods for analysing talk, text and interaction. London: Thousand Oaks, 2001.

35 Silver SA, Saragosa M, Adhikari NK, et al. What insights do patients and caregivers have on acute kidney injury and posthospitalisation care? a single-centre qualitative study from Toronto, Canada. BMJ Open 2018;8:e021418.

36 NHS England, NHS Improvement. The NHS patient safety strategy safer culture, safer systems, safer patients, 2019.

37 Murray J, Hardicre N, Birks Y, et al. How older people enact care involvement during transition from hospital to home: a systematic review and model. Health Expect 2019;22:883-93.

38 Armstrong D. Diagnosis and nosology in primary care. Soc Sci Med 2011;73:801-7.

39 Jutel A, Nettleton S. Towards a sociology of diagnosis: reflections and opportunities. Soc Sci Med 2011;73:793-800.

40 Bury M. Illness narratives: fact or fiction? Sociol Health IIIn 2001;23:263-85.

41 Bury M. Chronic illness as biographical disruption. Sociol Health IIIn 1982;4:167-82.

42 Cresswell KM, Sadler S, Rodgers S, et al. An embedded longitudinal multi-faceted qualitative evaluation of a complex cluster randomized controlled trial aiming to reduce clinically important errors in medicines management in general practice. Trials 2012;13:78.

43 Kreindler SA, Dowd DA, Dana Star N, et al. Silos and social identity: the social identity approach as a framework for understanding and overcoming divisions in health care. Milbank Q 2012;90:347-74.

44 Ling T, Brereton L, Conklin A, et al. Barriers and facilitators to integrating care: experiences from the english integrated care pilots. Int J Integr Care 2012;12:e129.

45 Elvey R, Bailey S, Checkland K, et al. Implementing new care models: learning from the greater Manchester demonstrator pilot experience. BMC Fam Pract 2018;19:89.

46 Nelson PA, Bradley F, Martindale A-M, et al. Skill-mix change in general practice: a qualitative comparison of three 'new' non-medical roles in English primary care. $\mathrm{Br} J$ Gen Pract 2019;69:e489-98.

47 Martindale A-M, Elvey R, Howard SJ, et al. Understanding the implementation of 'sick day guidance' to prevent acute kidney injury across a primary care setting in England: a qualitative evaluation. BMJ Open 2017;7:e017241.

48 McNab D, Bowie P, Ross A, et al. Systematic review and metaanalysis of the effectiveness of pharmacist-led medication reconciliation in the community after hospital discharge. BMJ Qual Saf 2018;27:308-20.

49 Think Kidneys. When or if to re-start ACEI, ARB, diuretics and other antihypertensive drugs after an episode of acute kidney injury, 2016.

50 Ahmad T, Jackson K, Rao VS, et al. Worsening renal function in patients with acute heart failure undergoing aggressive diuresis is not associated with tubular injury. Circulation 2018;137:2016-28.

51 Testani JM, Kimmel SE, Dries DL, et al. Prognostic importance of early worsening renal function after initiation of angiotensinconverting enzyme inhibitor therapy in patients with cardiac dysfunction. Circ Heart Fail 2011;4:685-91.

52 Cochrane Library. Disease management interventions for heart failure. Cochrane Database Syst Rev 2019.

53 Blum MR, Øien H, Carmichael HL, et al. Cost-Effectiveness of transitional care services after hospitalization with heart failure. Ann Intern Med 2020;172:248-57.

54 NHS England. Investment and evolution: a five-year framework for GP contract reform to implement the NHS long term plan. England: NHS, 2019.

55 Kellogg KM, Hettinger Z, Shah M, et al. Our current approach to root cause analysis: is it contributing to our failure to improve patient safety? BMJ Qual Saf 2017:26:381-387.

56 McKay J, Bradley N, Lough M, et al. A review of significant events analysed in general practice: implications for the quality and safety of patient care. BMC Fam Pract 2009:10:61

57 National Advisory Group on the Safety of Patients in England. A promise to learn - a commitment to act. improving the safety of patients in England: department of health, 2013. Available: https:// www.gov.uk/government/publications/berwick-review-into-patientsafety [Accessed 17 Oct 2019]. 
58 Spencer RA, Rodgers S, Salema N, et al. Processing discharge summaries in general practice: a qualitative interview study with GPs and practice managers. BJGP Open 2019;3

59 Phipps DL, Morris RL, Blakeman T, et al. What is involved in medicines management across care boundaries? a qualitative study of healthcare practitioners' experiences in the case of acute kidney injury. BMJ Open 2017;7:e011765.

60 Brown B, Gude WT, Blakeman T, et al. Clinical performance feedback intervention theory (CP-FIT): a new theory for designing, implementing, and evaluating feedback in health care based on a systematic review and meta-synthesis of qualitative research. Implement Sci 2019;14:40

61 Bailey S, Hunt C, Brisley A, et al. Implementation of clinical decision support to manage acute kidney injury in seconday care: an ethnographic study. BMJ Qual Saf 2019;0:1-8.

62 Logan R, Davey P, De Souza N, et al. Assessing the accuracy of ICD10 coding for measuring rates of and mortality from acute kidney injury and the impact of electronic alerts: an observational cohort study. Clin Kidney J 2019;3. 